\title{
GETTING LUCKY WITH ADAPTIVE OPTICS: FAST ADAPTIVE OPTICS IMAGE SELECTION IN THE VISIBLE WITH A LARGE TELESCOPE
}

\author{
N. M. LaW ${ }^{1}$, C. D. Mackay ${ }^{2}$, R. G. Dekany ${ }^{1}$, M. Ireland ${ }^{1,3}$, J. P. Lloyd ${ }^{4}$, A. M. Moore ${ }^{1}$, J. G. Robertson ${ }^{3}$, P. Tuthill ${ }^{3}$, \\ AND H. C. WOODRUFF ${ }^{3}$ \\ ${ }^{1}$ Department of Astronomy, Mail Code 105-24, California Institute of Technology, 1200 East California Blvd., Pasadena, CA 91125, USA; nlaw@astro.caltech.edu \\ ${ }^{2}$ Institute of Astronomy, University of Cambridge, Madingley Road, Cambridge CB3 OHA, UK \\ ${ }^{3}$ School of Physics, University of Sydney, NSW 2006, Australia \\ ${ }^{4}$ Department of Astronomy, Cornell University, Ithaca, NY 14853, USA \\ Received 2008 May 13; accepted 2008 October 21; published 2009 February 20
}

\begin{abstract}
We describe the results from a new instrument which combines Lucky Imaging and adaptive optics (AO) to give the first routine direct diffraction-limited imaging in the visible on a $5 \mathrm{~m}$ telescope. With fast image selection and alignment behind the Palomar AO system we obtained Strehl ratios of 5\%-20\% at $700 \mathrm{~nm}$ in a typical range of seeing conditions, with a median Strehl of approximately $12 \%$ when $10 \%$ of the input frames are selected. At wavelengths around $700 \mathrm{~nm}$ the system gave diffraction-limited 35 mas full width at half-maxima (FWHMs). At $950 \mathrm{~nm}$ the output Strehl ratio was as high as $36 \%$ and at $500 \mathrm{~nm}$ the FWHM resolution was as small as 42 mas, with a low Strehl ratio but with resolution improved by a factor of $\sim 20$ compared to the prevailing seeing. To obtain wider fields we also used multiple Lucky Imaging guide stars in a configuration similar to a ground layer AO system. With eight guide stars but very undersampled data we obtained 300 mas resolution across a $30^{\prime \prime} \times 30^{\prime \prime}$ field of view in the $i^{\prime}$ band.
\end{abstract}

Key words: instrumentation: adaptive optics - instrumentation: high angular resolution - methods: data analysis techniques: high angular resolution - techniques: image processing

Online-only material: color figures

\section{INTRODUCTION}

Adaptive optics (AO) has been used successfully for highangular-resolution imaging in the near-infrared (NIR) on a large number of 5-10 m class telescopes. However, it has not yet demonstrated routine diffraction-limited imaging in the visible on large telescopes. ${ }^{5}$ In this paper we describe the first system capable of producing diffraction-limited moderateStrehl images in the visible on a $5 \mathrm{~m}$ class telescope. The system combines the Lucky-Imaging (Fried 1978; Baldwin et al. 2001; Law et al. 2006b) frame selection and alignment concept and $\mathrm{AO}$ to produce much better performance than provided by either alone.

Direct Lucky Imaging has demonstrated routine diffractionlimited performance in the $I$ band on $2.5 \mathrm{~m}$ class telescopes (Mackay et al. 2004b; Law et al. 2005, 2006a, 2006b, 2008). A simple, low-cost system has been developed by the Cambridge Lucky Imaging group and similar systems have now been deployed by other groups (e.g., Hormuth 2007). Lucky Imaging, in contrast to speckle imaging, provides direct images which can be easily produced irrespective of the complexity of the science target.

We here describe the performance of visible light Lucky Imaging behind an $\mathrm{AO}$ system on a large telescope (the experiment is hereafter referred to as Lucky $+\mathrm{AO}$ ). We used the LAMP (LuckyCam, AO, aperture Masking and polarimetry at Palomar) instrument, an electron multiplying (EM) CCDbased camera custom-built for this experiment to operate in conjunction with the Palomar AO system PALMAO. The instrument offered three observation modes: Lucky Imaging (fast shift-and-add alignment followed by frame selection),

\footnotetext{
5 Although see Wizinowich et al. (2000) for some Keck-II telescope very short-exposure experimental results at $850 \mathrm{~nm}$.
}

adaptive-optics assisted aperture masking, and high-contrast imaging polarimetry. Results from the latter two modes will be described in separate publications.

During six nights on the Palomar 200" Hale telescope the system routinely produced diffraction-limited resolution images with 5\%-20\% Strehl at $700 \mathrm{~nm}$ (Figure 1). In this paper, we characterize the performance of the system under a variety of conditions and wavelengths with a view to guide the design of more permanent Lucky $+\mathrm{AO}$ instruments.

In Section 2, we describe the observations and instrument setup. In Section 3, we detail the performance of the system and the efficacy of the combination of Lucky Imaging and AO in the low-Strehl regime. We also describe our experiments in using multiple guide stars to obtain a larger useful field of view. We conclude in Section 4.

\section{OBSERVATIONS AND INSTRUMENT SETUP}

LAMP was used in a six night run on the $5 \mathrm{~m}$ Palomar Hale telescope from 2007 July 2 to 2007 July 8 . Time equivalent to two nights was devoted to the Lucky Imaging mode described here, during which we observed a variety of both test and science targets. The Palomar DIMM-MASS unit (Thomsen et al. 2007) was used to record seeing parameters during our observations.

LAMP was placed behind the Palomar AO system PALMAO (Troy et al. 2000; Dekany et al. 1998). The system has 241 active actuators with $5 \mu \mathrm{m}$ stroke, and a $16 \times 16$ subaperture Shack-Hartmann wavefront sensor. The system operates at up to $2 \mathrm{kHz}$ and typically updated at more than $200 \mathrm{~Hz}$ rates during our observations. In median Palomar conditions ( $V$-band seeing $=1^{\prime \prime} .1$ ) the typical bright-star wavefront error is $220 \mathrm{~nm} \mathrm{rms}$. We replaced the usual wavefront sensor visible/IR dichroic with a 50:50 beamsplitter to send visible light to the science focus.

LAMP was used in place of the AO system's usual NIR imaging camera. We built a simple re-imaging camera to provide 

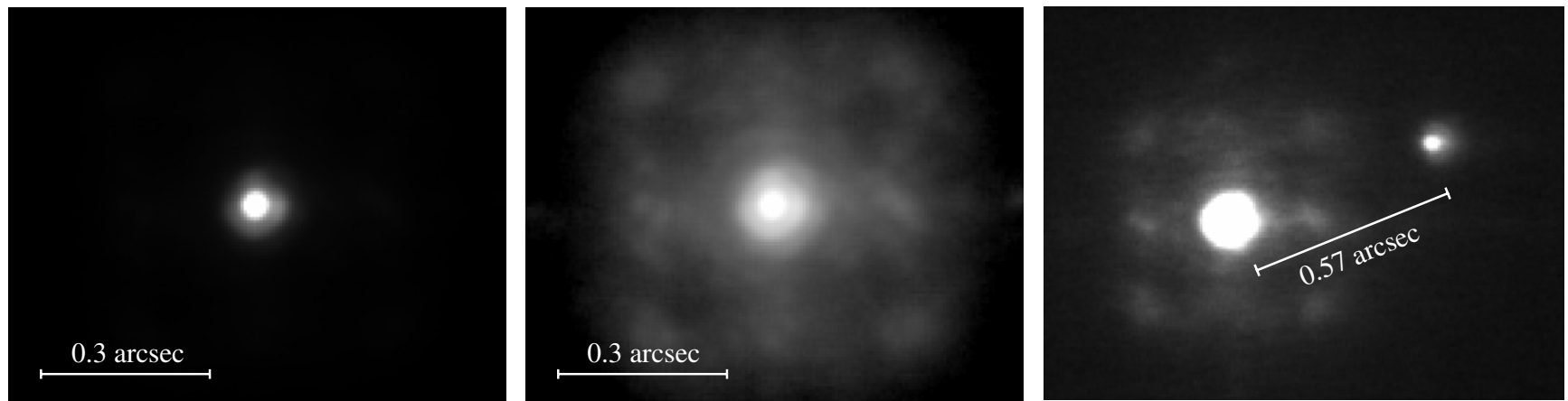

Figure 1. Examples of Lucky + AO point-spread functions (PSFs) acquired with LAMP at $710 \mathrm{~nm}$. Left: the single star HD 160507; a 5\% selection from a 600 s run at 20 frames per second (FPS; linear gray scale). Middle: the same image as the left panel but with a log scale. Right: the binary HD 235089 ( $\Delta m \sim 4.5,0$ '. 57 separation) observed at $710 \mathrm{~nm}$ ( $10 \%$ selection from $320 \mathrm{~s}$ at 50 FPS $)$.
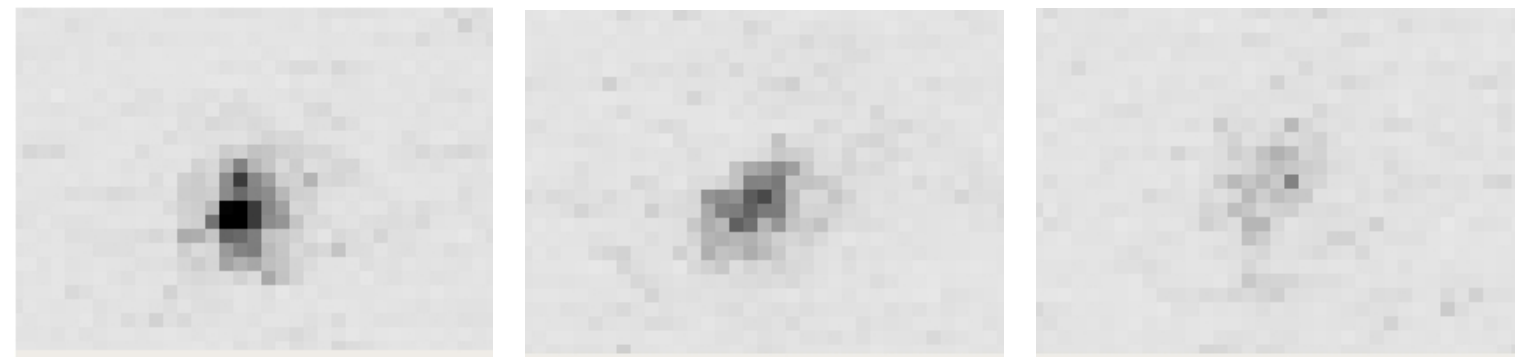

Figure 2. Frames selected from 6000 frames in a typical Lucky Imaging + AO run taken at 50 FPS at 710 nm with 14.9 mas pixels, targeted at the single star HD 192849. Left: one of the high-Strehl frames in the run. Middle: a frame with near-median Strehl. Right: one of the lowest-Strehl frames.

five field sizes ranging from $31^{\prime \prime}$ (61 mas pixels, undersampled $\sim 4 \times$ at $700 \mathrm{~nm}$ ) to 6.0 (Nyquist sampled at $500 \mathrm{~nm}$ ). An atmospheric dispersion corrector was employed to allow broadband observations.

The camera detector was based on an electron multiplying $528 \times 512$ E2V CCD97. The CCD's electron-multiplication process allows the detection of individual photons in each frame produced by the camera at the full quantum efficiency (up to $90 \%$ ) of the CCD (Mackay et al. 2004a). Our custom camera electronics are capable of running at up to 20 FPS in full $528 \times 512$ pixel frames; subarray readouts were used to increase the speed up to 50 FPS for some targets. The EMCCD gain was optimized for each target and ranged from 1 (no gain) to $\sim 10,000$. The camera produced 14-bit data at 7.5 megapixels per second. The data were recorded using custom software (Law 2007); a lossless compression algorithm reduced the data transfer requirements by an average factor of 1.9, allowing direct recording onto external USB hard disks.

We used the standard Lucky Imaging data reduction pipeline without modification; complete descriptions of the reduction process can be found in Law (2007) and Law et al. (2006b). The data acquisition software was capable of displaying a realtimepreview of the Lucky Imaging output but the full data reduction was performed by a scripted process during daytime operations. Briefly, recorded frames were bias-corrected, flat-fielded and cosmic rays were removed. A bright star in the field was selected to serve as a Lucky-Imaging guide star (in these observations this was typically the AO guide star). The frames were sorted in order of the Strehl ratio and those that met a specific quality criterion were selected and aligned to produce a final highangular-resolution image.

For frame selection and alignment, we estimate the Strehl ratio and optimal shift position for each image by cross-correlating the instantaneous guide star PSF with a diffraction-limited PSF. The height of the peak of the resulting two-dimensional array gives the degree of correlation $(\approx$ the Strehl ratio) and the position of the peak gives the optimal shift position to align the brightest speckles of each image. We perform the image shift position calculations on an image cubic-spline subsampled by $4 \times 4$ to give the positions for sub-pixel image alignment using the Drizzle (Fruchter \& Hook 2002) algorithm. Where calculations require the total stellar flux to be measured we use apertures of at least $4^{\prime \prime}$ diameter; tests confirmed that the flux outside this radius has a negligible effect on the Strehl ratios measured here.

We targeted a variety of relatively bright $\left(m_{V}=6-10\right)$ stars for these observations to ensure that photon noise was a small contribution to any observed PSF variability (for our faintest observed stars we calculate that photon noise could contribute at most one-third of the observed Strehl ratio variability). Frame selection of images containing photon noise can bias full width at half-maximum (FWHM) measurements of the guide stars (Law 2007); to avoid this we have measured all FWHMs and image profiles using the secondary component of close binaries. Strehl ratios noted in the text were calculated by comparing the measured PSF with a theoretical diffraction-limited PSF (which takes into account the telescope central obstruction and the effects of pixel-grid alignment).

\section{PERFORMANCE}

Figure 2 compares three representative frames recorded within a single 1 minute run. It is clear that there are large image quality variations. Typical Strehl ratio histograms recorded by our instrument behind the AO system are shown in Figure 3. The histograms cover a representative range of seeings and frame rates for our data set; it can be immediately seen that the Strehl ratio variations behind the AO system are sufficient that large Strehl ratio improvements can be realized by using only the frames with the greatest Strehl ratios. 


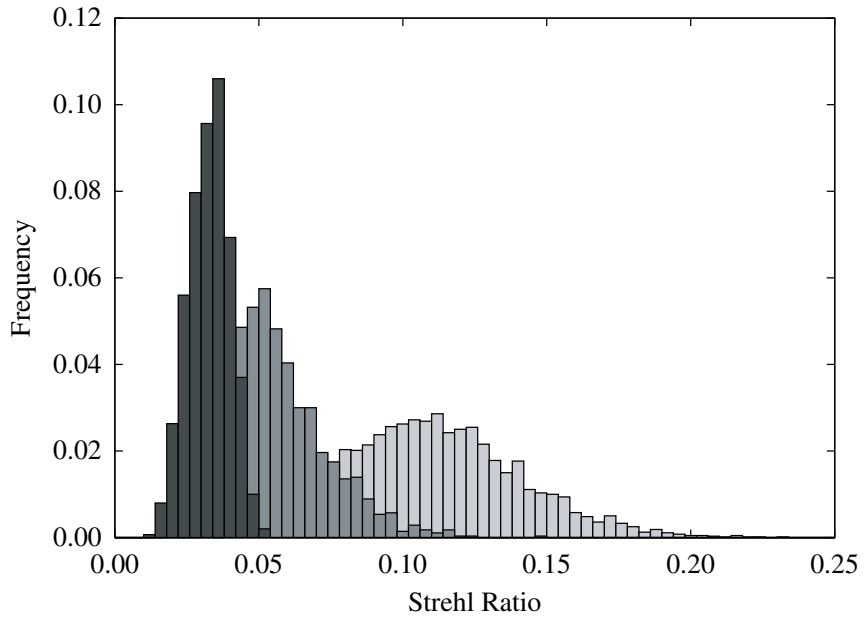

Figure 3. Strehl ratio histograms for three $710 \mathrm{~nm}$ wavelength observations from our data set with seeings ranging from 0.75 to $1{ }^{\prime \prime} 1$. Note that for clarity the darker histograms obscure parts of the lighter ones.

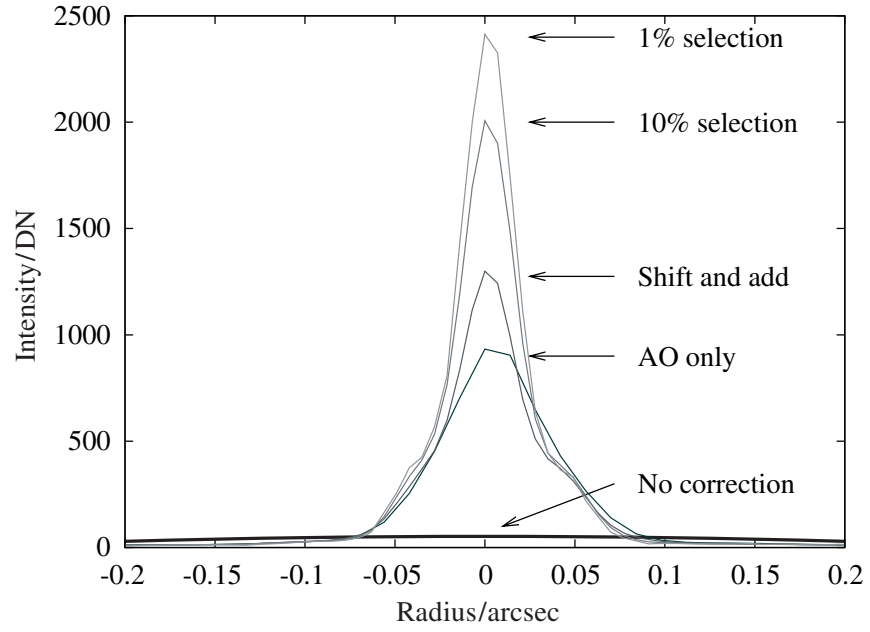

Figure 4. Cuts through a typical Lucky Imaging + AO PSF. The companion to HD 235089 was observed in $0{ }^{\prime \prime} 86$ seeing at 50 FPS in a $10 \mathrm{~nm}$ bandpass centered at $710 \mathrm{~nm}$, in a 3000 frame run. The $y$-axis is in data numbers as recorded by the camera. The "no correction" (seeing-limited) profile is modeled here by a Gaussian with the width measured from the Palomar DIMM during the observations and total flux equal to that of our $\mathrm{AO}$-corrected observations.

Each of the Strehl ratio distributions is positively skewed, increasing the fraction of high-Strehl outliers compared with a Gaussian. The Strehl variability behavior in this low-tomoderate Strehl regime contrasts with the negatively skewed distributions measured in the high-Strehl regime by Gladysz et al. (2006, 2008). Our Strehl ratio distributions appear similar to the behavior observed for fast-frame-rate observations without an AO system (Tubbs et al. 2002; Law 2007; Baldwin et al. 2008). The Gladysz results were obtained in the $K$-band on relatively small telescopes, with Strehl ratios up to $70 \%$ and are thus in a very different image quality regime from our data. As noted in Gladysz et al. (2006), in the low-Strehl regime covering the Lucky + AO experiment we would expect the observed positively-skewed Strehl ratio distributions.

\subsection{Single Star PSFs}

Figure 4 shows cuts through the output images from a typical Lucky Imaging + AO run. The Airy ring only becomes visible in the PSF cuts after frame selection and alignment; frame

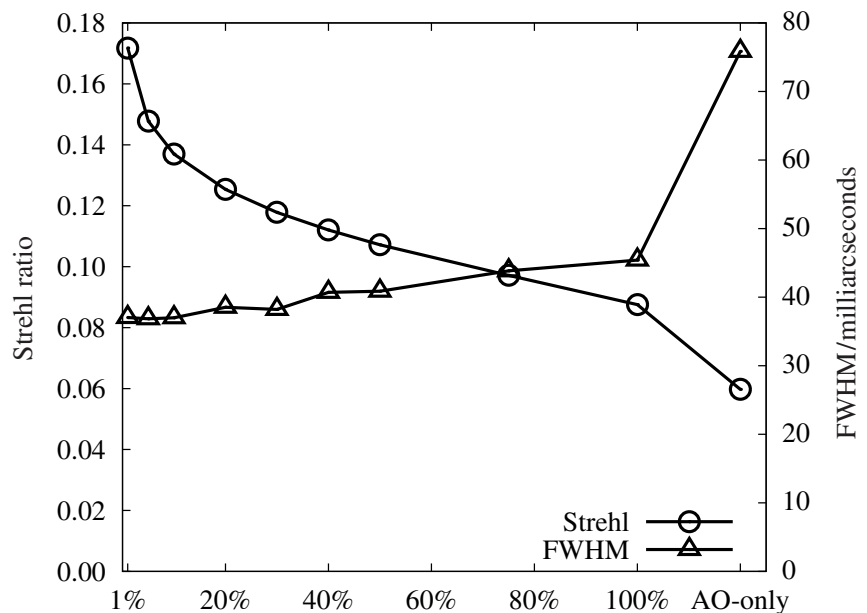

Figure 5. Strehl ratio and FWHM resolution as a function of frame selection percentage, for the same run as Figure 4. The 50 FPS run had a duration of $320 \mathrm{~s}$ during which the minute-timescale seeing was 0 ".86 with a peak-to-peak variability of 0.05 . AO-only is the result of a long-exposure (summing all the recorded frames) behind the AO system. 100\% "selection" is equivalent to shift-and-add using all the frames. Note that the data were slightly spatially undersampled, so the best resolution achievable for a diffraction-limited image is about 35 mas.

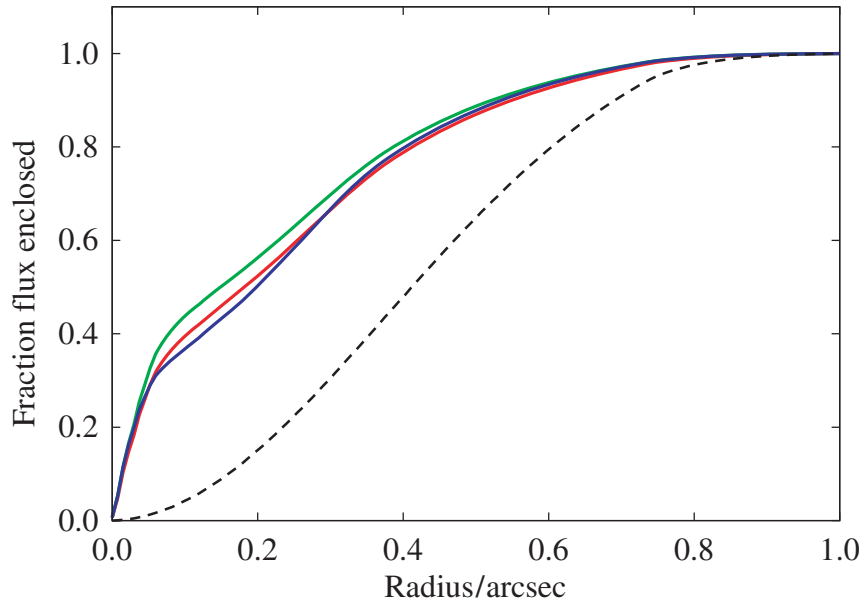

Figure 6. Enclosed fraction of total starlight as a function of circular aperture radius. Three representative runs are shown (upper lines), all were 10\% Lucky + AO selections from 320 s runs on HD 192849 and HD 235089 taken at 50 FPS in a $10 \mathrm{~nm}$ bandpass at $710 \mathrm{~nm}$; the seeing was $0 . .8-1{ }^{\prime \prime} 0$. The dashed line is a seeing-limited profile modeled by a Gaussian with 0.9 FWHM. Note that the structure of all the Lucky + AO curves is very similar, and the $50 \%$ enclosed flux radius is less than 0 .' 2 .

(A color version of this figure is available in the online journal.)

selection leads to an improved Strehl ratio and a decreased FWHM. This is quantified for a typical run in Figure 5. Using $20 \%$ of the frames Lucky Imaging reached diffraction-limited resolution for the telescope and provided a more than $2 \times$ improvement in the FWHM and Strehl ratio compared to using the AO system alone. Further improvements in the Strehl ratio were achieved with more stringent frame selections, while the FWHM remained diffraction-limited. Note that the more stringent selections concentrate light from the profile wings visible in Figure 1 and so appear above the unselected PSF at all radii shown in Figure 4. Figure 6 details the enclosed flux as a function of the circular aperture radius for three example Lucky + AO runs.

Under the conditions considered here, the Strehl ratio increases by a factor of 2 as the quality criterion is made more 


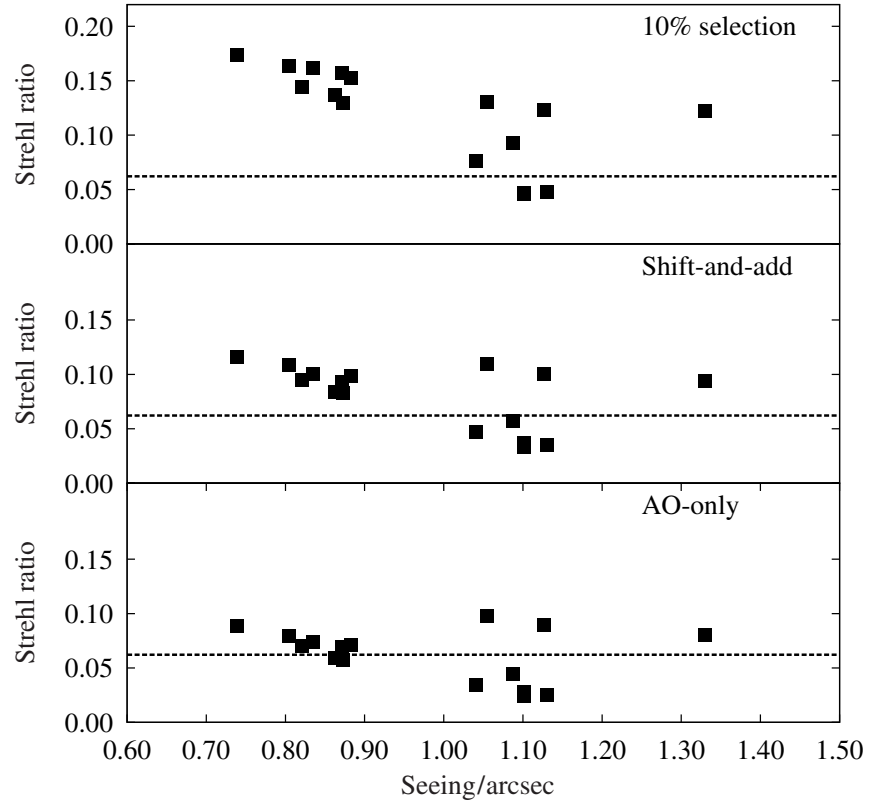

Figure 7. Strehl ratio as a function of seeing. Bottom panel: long-exposure AO imaging (averaging the frames recorded by the camera). Top panel: the results of Lucky Imaging of the AO data, selecting $10 \%$ of the frames. To guide the eye, the dashed line in each panel shows the average Strehl ratio achieved by the long-exposure AO imaging. To obtain a wide range of seeing, the data presented here were taken over several hours on two separate nights. Each data point is a 1.0-4.0 min data set taken at frame rates between 20 and 50 FPS. Data were recorded in $10 \mathrm{~nm}$ passbands centered at $670 \mathrm{~nm}$ or $710 \mathrm{~nm}$; the images were sampled at 14.9 mas pixel $^{-1}$.

stringent. The FWHM, however, is almost diffraction-limited even with only shift-and-add alignment and no frame selection (Figure 5). Frame alignment reduces the FWHM in size by almost a factor of 2 , suggesting that a large component of the residual wavefront error produced by the AO system is in tip/tilt. However, we note that we guide on the brightest speckle rather than the centroid of the image, and so in the lowStrehl/multiple-speckle regime we obtain much higher quality PSFs than centroiding tip-tilt correction can provide (Christou 1991). This is evident in the measured PSF positions in our images: in typical observations the rms centroid motion is approximately 8 mas, while the rms PSF core motion is approximately 18 mas.

Figure 7 details the performance under a range of seeing conditions. In all observations Lucky $+\mathrm{AO}$ significantly improved on the performance of $\mathrm{AO}$ alone. Under median Palomar Observatory seeing (1".1) and selecting $10 \%$ of the frames Lucky + AO gave output Strehl ratios of 0.05 to 0.13 at $700 \mathrm{~nm}$. In 0.7 seeing the performance increased to $17 \%$ Strehl ratios. In all cases the system gave an improvement in Strehl of between $2 \times$ and $3 \times$ compared to the PALMAO system alone.

\subsection{Companion Detection}

The benefits of the results described above are clear for crowded field data, where simply increasing the separation of stars through high-angular resolution is most important. In this section we investigate the optimization of the Lucky Imaging process for a faint, close stellar companion detection.

As the frame selection criterion is made more stringent the light in the PSF becomes more concentrated. This affects both the background against which a faint companion must

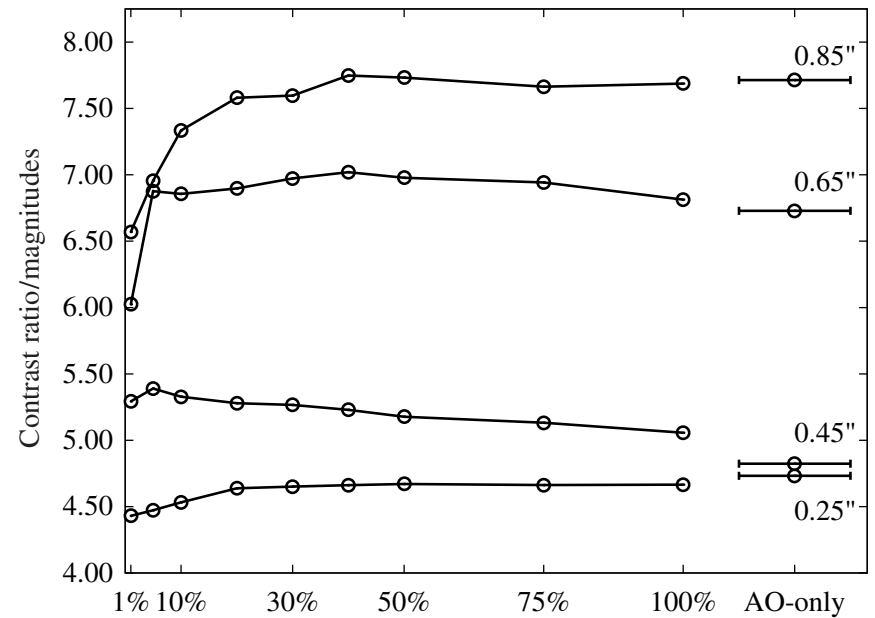

Figure 8. Companion detection magnitudes. HD 235089, a 0.'57 binary observed at $710 \mathrm{~nm}, 50$ FPS for $320 \mathrm{~s}$ is used to calculate the limiting contrast ratio at which a companion can be detected with a signal-to-noise ratio $(\mathrm{S} / \mathrm{N})$ of 10 . The specified binary separation is noted at the right of each line. The PSF of the real companion was used to determine the signal within a six-pixel aperture. For each radius the rms noise and its variance was measured at eight apertures distributed around the primary. We conservatively set the effective background noise at that radius to be a $2 \sigma$ excursion above the average rms noise. The bars at the right of the plot give the long-exposure performance behind the AO system, using the same data.

be detected and the amplitude of its signal within a diffractionlimited core. However, the smaller effective integration time also increases the signal and background noise. The tradeoff between these effects is described in Figure 8 where we show measured companion detection limits for a range of close companions versus the frame selection level for a typical observation (based on the binary shown in the right panel of Figure 1).

There is little or no drop in faint companion detection performance with frame selections as stringent as $10 \%$, at all tested radii. At the smallest separations ( $\lesssim 0^{\prime \prime} 1$, not shown), Lucky image selection is required to resolve the binaries. These results demonstrate that, compared to long-exposure AO imaging, Lucky + AO close companion detection can be performed with higher angular resolution and with minimal $\mathrm{S} / \mathrm{N}$ performance loss.

\subsection{Performance at Other Wavelengths}

To investigate the performance of Lucky $+\mathrm{AO}$ as a function of wavelength we observed HD 235089 in a set of bands between 500 and $950 \mathrm{~nm}$ (Figure 9). The observations were taken during a 45 minute period during which the DIMM seeing was $0 . \prime 80 \pm 0.15$. The wavelength range was limited in the blue by the lower throughput of the AO system optics, and in the red by our CCD detector's sensitivity. As above, we use the star's companion (0'.6 separation) to avoid biasing the PSF shape by the influence of photon noise on frame selection.

In all cases the output FWHM resolution was improved by a factor of at least 2 compared to the capabilities of the AO system alone. At $500 \mathrm{~nm}$ the resolution achieved was as good as 43 mas, not diffraction-limited but still a factor of 20 improvement over the natural seeing.

As would be expected, the system gives a decreased output Strehl ratio at shorter wavelengths. However, the fractional increase in the Strehl ratio is actually greater at $500 \mathrm{~nm}$ than at $710 \mathrm{~nm}$, and the frame-selection process gives the most obvious FWHM improvements at $500 \mathrm{~nm}$. It seems that there is an 

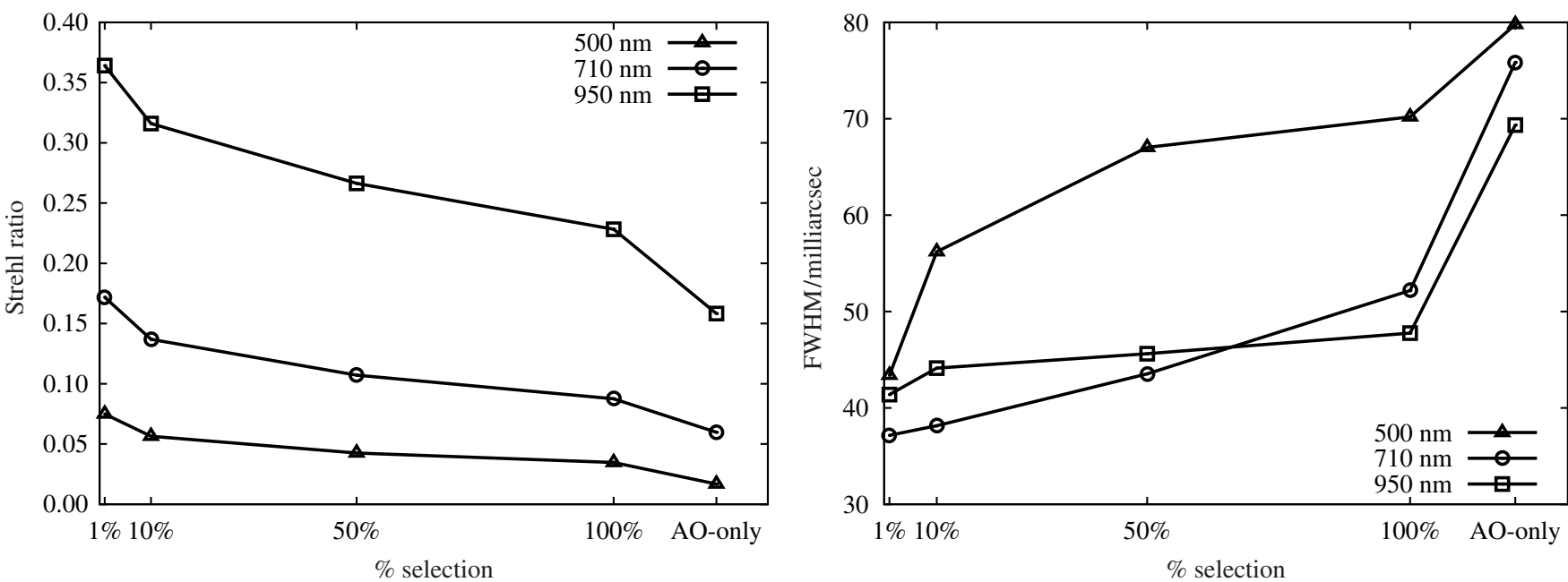

Figure 9. Strehl ratio and FWHM as a function of wavelength and number of frames selected. Each $320 \mathrm{~s}$ observation was taken at 50 FPS in a $10 \mathrm{~nm}$ bandpass filter As in Section 3.1, to avoid biasing the FWHM measurement, the companion of HD 235089 was used as the performance measurement star while the AO and Lucky Imaging systems guided on the primary. Note that the increased FWHM of the Lucky Imaging output PSFs at $950 \mathrm{~nm}$ compared to $700 \mathrm{~nm}$ is due to the increased diffraction-limited core size at those wavelengths. Slight undersampling of the original PSFs gives a slightly increased FWHM compared to that naively expected for a diffraction-limited PSF on a $5 \mathrm{~m}$ telescope. $100 \%$ selection is equivalent to shift-and-add brightest speckle alignment for all frames.

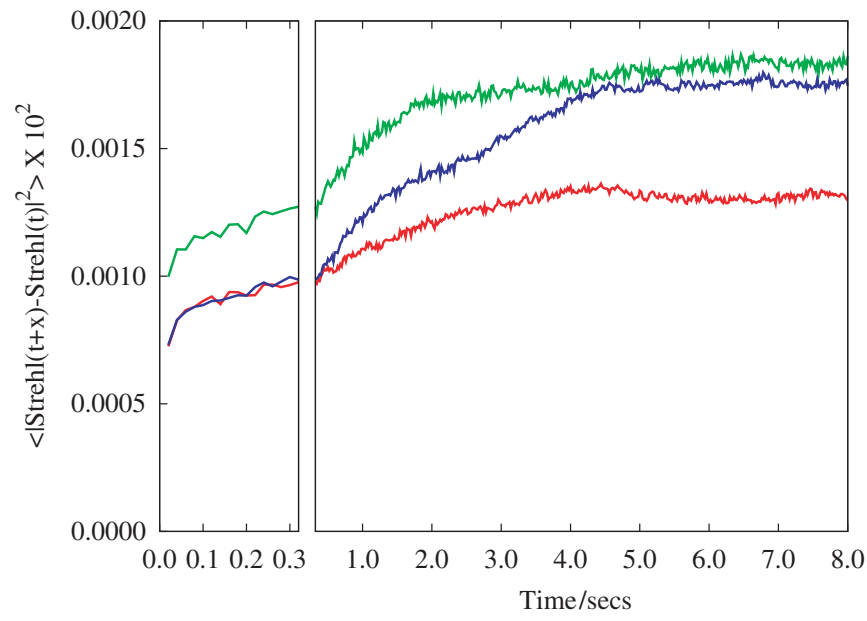

Figure 10. Structure functions, the typical Strehl ratio variance between frames as a function of time, for three typical $320 \mathrm{~s}$ runs taken at 50 FPS at $710 \mathrm{~nm}$ in a $10 \mathrm{~nm}$ bandpass. Note the slight downturn at the minimum frame spacing of $0.02 \mathrm{~s}$, suggesting that we are approaching but not reaching the coherence time.

(A color version of this figure is available in the online journal.)

increased frame sharpness variance associated with the lowerquality images at shorter wavelengths.

\subsection{Frame Rates}

Figure 10 gives the Strehl ratio structure functions for three typical observations. It is clear from these measurements that at our highest speed of 50 FPS the instrument is not completely sampling the PSF changes behind the AO system, with most of the observed variation occurring on timescales on the level of single frames. For stars of the brightness used here, the photon and EMCCD multiplication noise accounts for at most onethird of the observed single-frame-time variations in Strehl; it appears that we really are significantly undersampling the true behind-AO Strehl ratio variations. This might be expected given the hundreds of FPS rates typically employed by the PALMAO system for turbulence correction. We note that future Lucky + AO systems may wish to provide 100+ FPS to achieve the highest possible Strehl ratios.

\subsection{Isoplanatic Patch}

In the above sections we used the $\mathrm{AO}$ guide star as the Lucky Imaging guide star; the greater guide-star sensitivity of Lucky Imaging leads the AO guide star to always be bright enough for Lucky guiding. If there are multiple guide stars in the field, however, we can investigate more complex frame quality-sensing strategies. In this experiment, we use multiple guide stars in a $30^{\prime \prime}$ field in the core of the M13 globular cluster.

To obtain a wide field of view we had to use very spatially and temporally undersampled data (60 mas pixels, 20 FPS). The performance, while much better than the prevailing 0'.60 seeing, is therefore greatly decreased compared to the results described earlier and what could be achieved with a faster, larger array camera system.

When operating the data reduction system in the usual mode of using the AO guide star as the Lucky Imaging reference, the FWHM approximately doubles over a $15^{\prime \prime}$ radius (Figure 11, left panel). A very different behavior is observed when a different star is used for Lucky Imaging guiding (Figure 11, middle panel). The performance around the Lucky Imaging guide star is greatly improved, from 400 mas FWHM to 240 mas FWHM, while the performance around the AO guide star is reduced. Guiding on the new star caused different frames to be selected as well as different image shifts to be used; the system is not simply correcting tip/tilt anisoplanatism. It appears that the AO system occasionally "Luckily" produced corrections that improved the turbulence experienced in other parts of the image.

The possibility of correcting different areas of the field with different selections of the same data suggests a method of improving the isoplanatic patch if multiple guide stars are available, in a setup similar to a multiple-guide-star ground layer AO (GLAO) system. Using eight Lucky guide stars simultaneously, and selecting the optimal guide star to use for each point in the image, the entire $30^{\prime \prime} \times 30^{\prime \prime}$ field can be processed to give FWHM resolutions better than 300 mas in the $I$ band (Figure 11, right panel).

\section{DISCUSSION AND CONCLUSIONS}

The combination of Lucky Imaging and $\mathrm{AO}$ produced 35 mas resolution at $700 \mathrm{~nm}$ on the Palomar $200^{\prime \prime}$ telescope, 

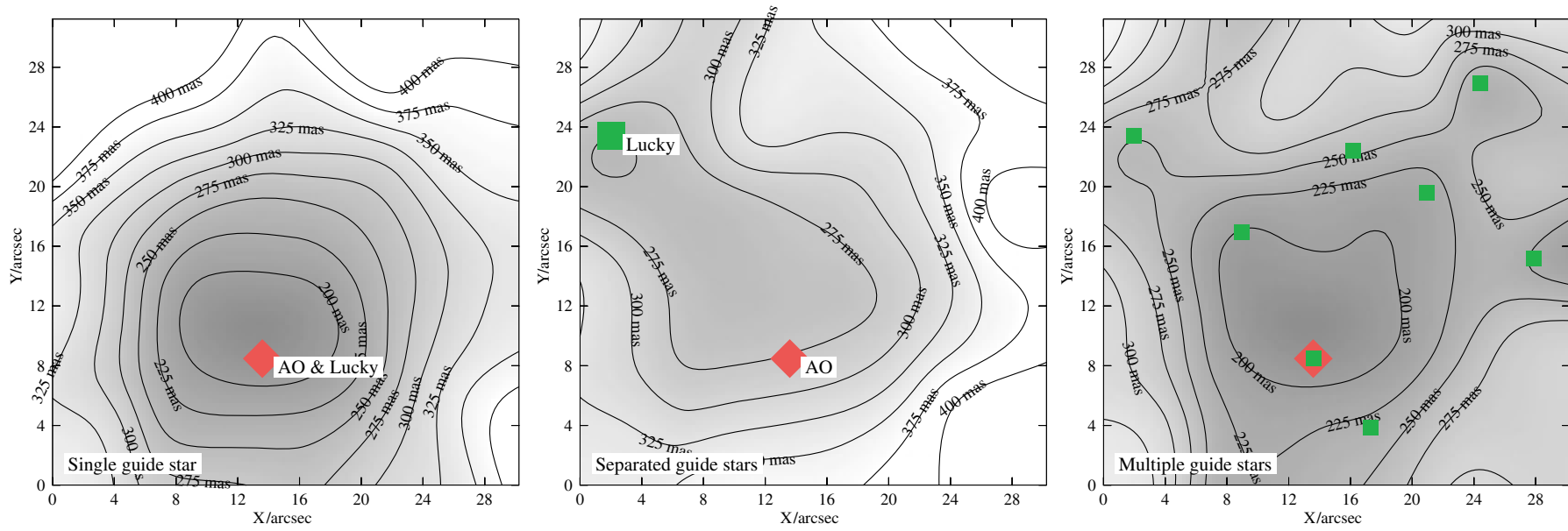

Figure 11. Maps of the variation in FWHM over a $30^{\prime \prime}$ field in the core of the M13 globular cluster, taken with severely undersampled pixels in the SDSS $i^{\prime}$ filter (770 $\mathrm{nm}$ central wavelength). The gray scale is set to the same levels in each image for comparison; the variation in FWHM is measured from the profiles of 74 stars distributed throughout the field. Left: Lucky Imaging on the AO guide star. Middle: Lucky Imaging on a different guide star. Note the change in the variation of the image resolution, becoming elongated between the Lucky Imaging and AO guide stars. Right: a map constructed from the same data using eight different Lucky-Imaging guide stars simultaneously and selecting the optimal Lucky guide star for each point in the image.

(A color version of this figure is available in the online journal.)

extending the useful wavelength range of the Palomar AO system into the visible. Near-diffraction-limited resolution was achieved with shift-and-add frame alignment alone, which may itself be a very useful capability for a variety of science programs. With the combination of frame selection and alignment the diffraction limit and Strehl ratios more than $10 \%$ were obtained.

The performance detailed in the above sections is unlikely to be the best achievable with systems of this type. Our results suggest that such systems should run very rapidly, as even 50 FPS observations undersampled the Strehl ratio variations. In addition, some of our observations are of lower performance than would be expected for the prevailing seeing conditions (Figure 7). This may be due to insufficiently precise non-common-path (NCP) error calibrations on some nights. Although the Palomar AO system has demonstrated NCP calibrations as precise as $34 \mathrm{~nm}$ wavefront error with the standard NIR camera, LAMP typically achieved a Strehl ratio of only $\sim 30 \%$ on PALMAO's built-in turbulence-free white light source in the visible. Future Lucky $+\mathrm{AO}$ instruments will likely obtain higher performance as better NCP error calibration is achieved.

Although the observations described here were of relatively bright $\left(m_{V}=6-10\right)$ stars, we note that the system is quite capable of guiding on much fainter stars. Lucky Imaging (without an AO system) can guide on stars as faint as 16th magnitude (Law et al. 2006b) on $2.5 \mathrm{~m}$ telescopes and the Lucky $+\mathrm{AO}$ technique is therefore capable of using any guide star that is bright enough for adaptive-optics operation (assuming broadband imaging and half the light going to the $\mathrm{AO}$ wavefront sensor). Laser guide star extensions to the Lucky + AO technique will require techniques to mitigate the extra background from the laser backscatter, but are possible in principle.

As the first system enabling diffraction-limited moderateStrehl performance in the visible on $5 \mathrm{~m}$ class telescopes, the science applications are broad. In particular, crowded field visible photometry (for example, in extragalactic resolved stellar population studies) and high-angular-resolution studies of nebular emission lines in the visible will benefit greatly from the increased resolution.
The enlarged useful field of view possible with multiple guide stars may greatly improve the range and size of fields which can be covered. We recommend further investigation of the performance of this mode with higher frame rate cameras and improved PSF sampling.

The seeing during our experiments was typical for the Palomar Observatory site, mostly around $1{ }^{\prime \prime}$. This offers the intriguing possibility that these resolutions could be improved using a larger telescope in better seeing conditions-for example, the Keck telescope in 0'.5 seeing may be capable of reaching its diffraction limit in the visible using Lucky + AO techniques.

The combination of Lucky Imaging and AO offers a relatively simple, low-cost upgrade to current AO systems. As the first direct diffraction-limited imaging in the visible on large telescopes this technique pioneers the science which will be performed with future visible-AO systems.

We particularly thank the PALMAO team, especially Jenny Roberts and Antonin Bouchez, for all their help during the design, setup, and operation of the instrument. Thanks also go to the Palomar Observatory team for great assistance throughout our run. We thank Chris Koresko for providing the ADC design. Facilities: Hale

\section{REFERENCES}

Baldwin, J. E., Tubbs, R. N., Cox, G. C., Mackay, C. D., Wilson, R. W., \& Andersen, M. I. 2001, A\&A, 368, L1

Baldwin, J. E., Warner, P. J., \& Mackay, C. D. 2008, A\&A, 480, 589

Christou, J. C. 1991, PASP, 103, 1040

Dekany, R. G., Brack, G., Palmer, D., Oppenheimer, B. R., Hayward, T. L., \& Brandl, B. 1998, Proc. SPIE, 3353, 56

Fried, D. L. 1978, Opt. Soc. Am. J. A, 68, 1651

Fruchter, A. S., \& Hook, R. N. 2002, PASP, 114, 144

Gladysz, S., Christou, J. C., Bradford, L. W., \& Roberts Jr., L. C. 2008, PASP, 120,1132

Gladysz, S., Christou, J. C., \& Redfern, M. 2006, Proc. SPIE, 6272, 62720J

Hormuth, F., Brandner, W., Hippler, S., Janson, M., \& Henning, T. 2007, A\&A, 463,707

Law, N. M. 2007, The Observatory, 127, 71

Law, N. M., Hodgkin, S. T., \& Mackay, C. D. 2006a, MNRAS, 368, 1917

Law, N. M., Hodgkin, S. T., \& Mackay, C. D. 2008, MNRAS, 384, 150 
Law, N. M., Hodgkin, S. T., Mackay, C. D., \& Baldwin, J. E. 2005, Astron. Nachr., 326, 1024

Law, N. M., Mackay, C. D., \& Baldwin, J. E. 2006b, A\&A, 446, 739

Mackay, C., Basden, A., \& Bridgeland, M. 2004a, Proc. SPIE, 5499, 203

Mackay, C. D., Baldwin, J., Law, N., \& Warner, P. 2004b, Proc. SPIE, 5492, 128
Thomsen, M., Britton, M., \& Pickles, A. 2007, in American Astronomical Society Meeting Abstracts 210, 117.01

Troy, M., et al. 2000, Proc. SPIE, 4007, 31

Tubbs, R. N., Baldwin, J. E., Mackay, C. D., \& Cox, G. C. 2002, A\&A, 387, L21

Wizinowich, P., et al. 2000, PASP, 112, 315 